\title{
Fabrication of IoT Force Sensor Module in Five-day Program for Students as Part of Nanotechnology Platform Japan Project
}

\author{
Kentaro Totsu, ${ }^{*}$ Masaaki Moriyama, Hiraku Watanabe, Toshiyuki Kikuta, \\ Masahiro Hemmi, Masaaki Shoji, Takashi Yoshida, and Masataka Tatsuta \\ Micro System Integration Center, Tohoku University, \\ 519-1176 Aramaki-Aza-Aoba, Aoba-ku, Sendai 980-0845, Japan
}

(Received April 3, 2019; accepted May 20, 2019)

Keywords: IoT, MEMS, piezoresistive sensor, WiFi, wireless

An IoT force sensor module was fabricated and demonstrated in a five-day practical program for students, as part of the Nanotechnology Platform Japan project. The module mainly consists of a silicon-based piezoresistive micro-electromechanical system (MEMS) force sensor and a microcontroller with a WiFi wireless communication function. The MEMS force sensor was fabricated on a 4-inch silicon wafer in four days by each student. The IoT force sensor module was connected to an IoT cloud server via the internet and was successfully monitored by a smartphone. All of the fabrication, implementation, and testing processes were carried out by students at Hands-On-Access Fabrication Facility, Tohoku University.

\section{Introduction}

The Nanotechnology Platform Japan project, sponsored by the Ministry of Education, Culture, Sports, Science and Technology (MEXT), offers a practical program for students every year. $^{(1)}$ Tohoku University, a member of the Nanotechnology Platform Japan project, produces a five-day program on micro-electromechanical system (MEMS) process technology or sensor fabrication annually. In 2018, the fabrication of a MEMS force sensor module for IoT application was adopted as the task. IoT is a promising application of MEMS technology and its demand and market are now rapidly increasing. To respond to this social demand for IoT and to offer a more practical program for students, we prepared a task combining MEMS sensor fabrication and IoT module implementation. The IoT sensor module mainly consists of a silicon-based piezoresistive MEMS force sensor and a microcontroller with a WiFi wireless communication function for the internet. The principle of the sensor is that the resistance of the piezoresistive element changes with the strain generated by an applied force. ${ }^{(2,3)}$ We consider that the fabrication of such a piezoresistive force sensor is suitable for students because the suggested fabrication process is relatively simpler than that of other major types of sensors, such as capacitive force sensors; capacitive force sensors normally require the bonding of two processed substrates with a certain gap to form a capacitor, ${ }^{(4)}$ which takes a long time.

*Corresponding author: e-mail: totsu@tohoku.ac.jp

https://doi.org/10.18494/SAM.2019.2390 
Furthermore, the fabrication process for piezoresistive elements includes typical processes for MEMS as well as semiconductors, including ion implantation and annealing. Piezoresistive sensors are one of the most popular MEMS devices and are widely used in commercial products, such as pressure sensors. ${ }^{(5)}$

In August 2018, three students from universities participated in this program and each student successfully fabricated an IoT force sensor module. The features of this program are the combination of silicon-based MEMS sensor fabrication in a clean room, parts assembly, software programming, and testing in five days. To complete the program in five days, a simplified fabrication process for the MEMS force sensor was developed. The entire process was carried out at Hands-on-Access Fabrication Facility, Jun-ichi Nishizawa Memorial Research Center, Tohoku University. ${ }^{(6,7)}$ This is an open access facility and is mainly equipped with tools for 4- and 6-inch MEMS and semiconductor development in a $1800 \mathrm{~m}^{2}$ clean room. The facility was opened in 2010 and has been utilized by more than 250 companies.

In this paper, we describe the structure and fabrication process of the MEMS force sensor, the implementation of the IoT sensor module, and an example of testing.

\section{Sensor Structure and Fabrication}

\subsection{Principle and structure of force sensor}

A schematic diagram of the piezoresistive sensor is shown in Fig. 1. The silicon-based piezoresistive sensor fabricated using MEMS technology is attached to a printed circuit board (PCB) by an adhesive. Force is applied to a diaphragm of about $100 \mu \mathrm{m}$ thickness through a rubber hemisphere. Four piezoresistive elements forming a Wheatstone bridge are arranged at the edge of the diaphragm to detect the maximum strain generated by the force ${ }^{(2,3)}$ For this force sensor, p-type piezoresistive elements are diffused into a (100)-plane n-type silicon

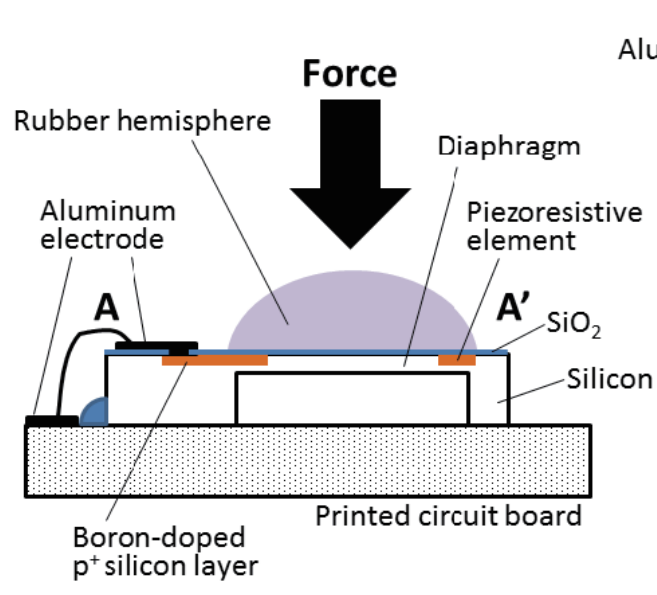

(a)

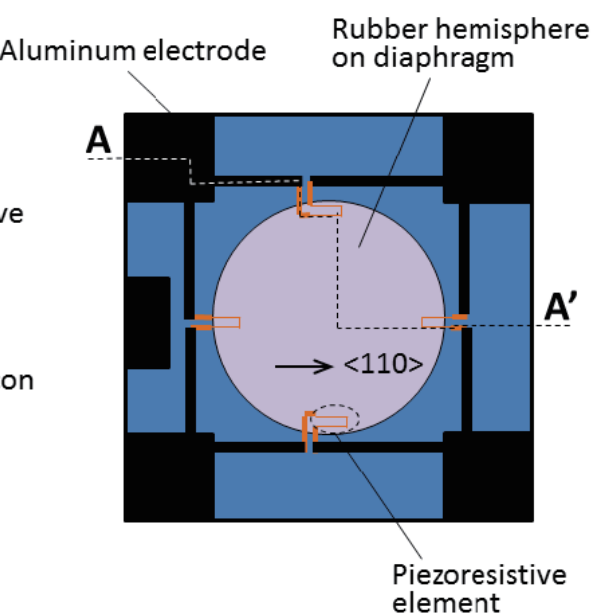

(b)

Fig. 1. (Color online) Schematic diagram of piezoresistive force sensor. (a) Cross section and (b) top view of the sensor. 
substrate. As the piezoresistivity coefficient in the $<110>$ direction is maximum in the (100) plane, ${ }^{(8)}$ all of the piezoresistive elements are oriented along the $<110>$ direction in parallel as shown in Fig. 1. When a force is applied to the diaphragm, one pair of facing piezoresistive elements is stressed longitudinally and the other pair is stressed transversely, resulting in the reversed polarity of the resistance change. By differential amplification at the output of the Wheatstone bridge, a change in applied force can be captured as a voltage signal. We use boron as a dopant, for which the target concentration is $2 \times 10^{20}$ atoms $/ \mathrm{cm}^{3}$. As sensor stability has priority over sensor sensitivity in this short-term program, we choose such a high doping concentration of the piezoresistive element to reduce the thermal coefficient of resistance and achieve an ohmic contact with the aluminum interconnection layer, even though the piezoresistivity coefficient is smaller than that at a lower concentration. ${ }^{(9)}$ The wide-area lowresistance silicon pattern is used for electrical connections between the piezoresistive elements and the aluminum patterns. The low-resistance silicon pattern is simultaneously realized with the boron doping of the piezoresistive elements. If more time is available for the fabrication, the doping concentrations can suitably and separately be chosen for the piezoresistive elements and the low-resistance silicon pattern. The thin-film aluminum pattern is in electrical contact with the low-resistance silicon pattern at the outside of the diaphragm and forms an electrode pad positioned at each corner of the sensor. The pads and electrodes on the PCB are connected by aluminum wires. The size of the sensor is $5 \times 5 \mathrm{~mm}^{2}$. The length and width of the piezoresistive element and the diameter of the diaphragm are varied from 100 to $600 \mu \mathrm{m}, 1$ to 5 $\mu \mathrm{m}$, and 1 to $3 \mathrm{~mm}$, respectively.

\subsection{Fabrication of force sensor}

The fabrication process flow of the piezoresistive MEMS sensor is depicted in Fig. 2. The schedule of each process in the program for students in August 2018 is also indicated in the

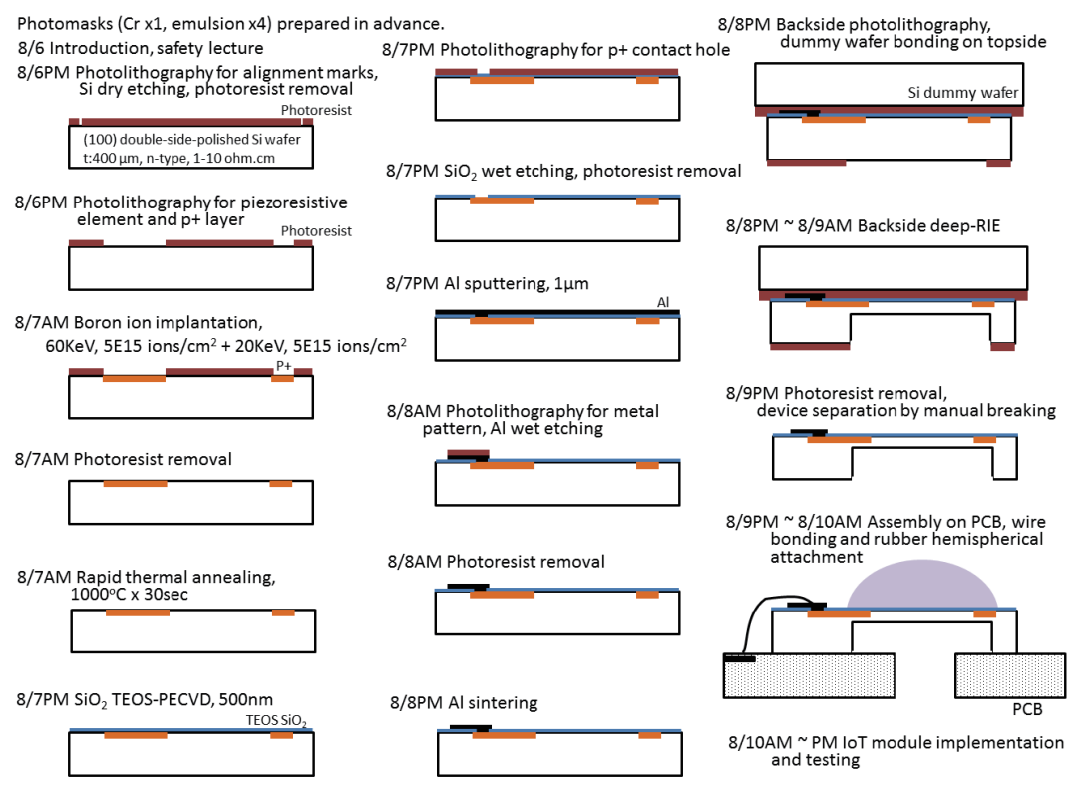

Fig. 2. (Color online) Fabrication process flow of piezoresistive force sensor. 
figure as follows: four days for sensor fabrication and one day for IoT module implementation and testing. We designed and fabricated the mask in advance. An n-type, 4-inch, (100)-plane, double-side-polished silicon wafer of $400 \mu \mathrm{m}$ thickness with $1-10 \mathrm{ohm} / \mathrm{cm}$ resistivity was given to each student. First, alignment marks for the subsequent photolithography process were prepared on the wafer using a reactive ion etching (RIE) tool (DEA-506, Anelva). The etching depth was about $200 \mathrm{~nm}$. A contact mask aligner (MA/BA6, SUSS MicroTec) was used for the entire photolithography process as shown in Fig. 3. Then, two-step boron ion implantation using an ion implanter (NH-20SR, Nissin) through the patterned photoresist as the mask was carried out to simultaneously form a $\mathrm{p}^{+}$layer for the piezoresistive elements and a low-resistance silicon pattern for electrical connection with the aluminum interconnection layer. The acceleration voltage and ion current of the ion implantation were $60 \mathrm{keV}$ and $5 \times 10^{15}$ ions $/ \mathrm{cm}^{2}$ in the first step and $20 \mathrm{keV}$ and $5 \times 10^{15}$ ions $/ \mathrm{cm}^{2}$ in the second step. After rapid thermal annealing for boron ion activation using a rapid thermal annealer (AG4100, AG Associates), a 500-nm-thick silicon dioxide film was deposited as a passivation layer using a tetraethoxysilane plasmaenhanced chemical vapor deposition (TEOS-PECVD) tool (MPX-CVD, Sumitomo Precision Products). Then, the silicon dioxide film was patterned to realize contact holes in the $\mathrm{p}^{+}$silicon layer. After sputtering 1- $\mu \mathrm{m}$-thick aluminum using a sputtering machine (i-Miller CFS-4EPLL, Shibaura Mechatronics), the aluminum layer was patterned by wet etching to form the electrode pads and the electrical interconnection between the pads and the $\mathrm{p}^{+}$layer, then the aluminum was sintered at $400{ }^{\circ} \mathrm{C}$ for $1 \mathrm{~h}$ in nitrogen. After patterning a thick photoresist on the back side of the wafer and bonding to a dummy wafer to support the processed wafer using the photoresist, deep reactive ion etching (DRIE) using a dry etcher (MUC-21, Sumitomo Precision Products) was carried out to form the diaphragm structure. The etching depth was about 300 $\mu \mathrm{m}$. A completely processed 4-inch wafer is shown in Fig. 4. Instead of carrying out a dicing process after the DRIE, device separation was performed by manually breaking the wafer along

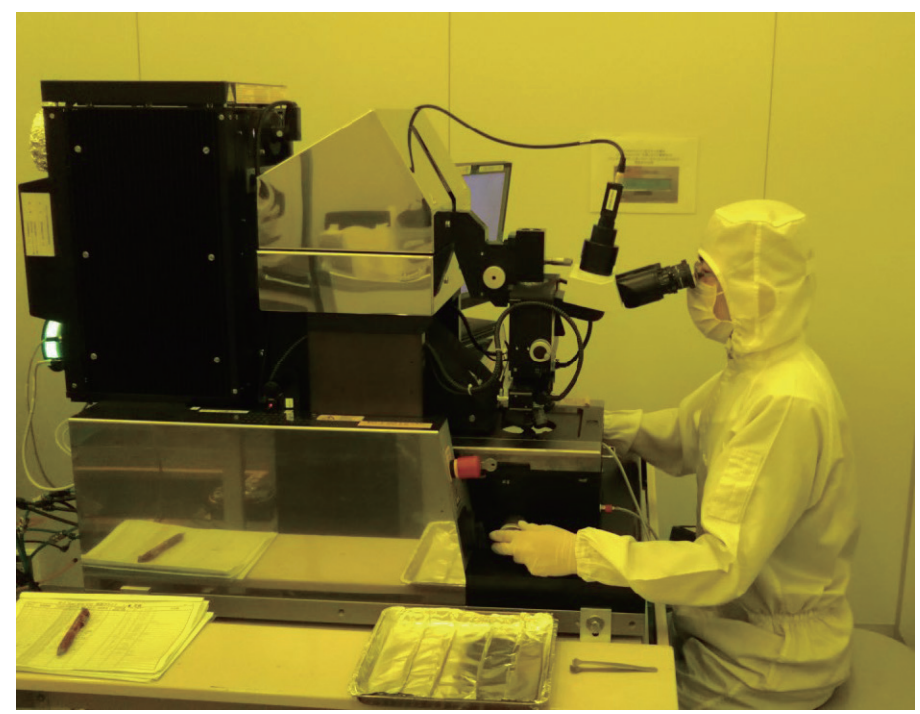

Fig. 3. (Color online) Alignment and exposure process using contact mask aligner operated by student. 


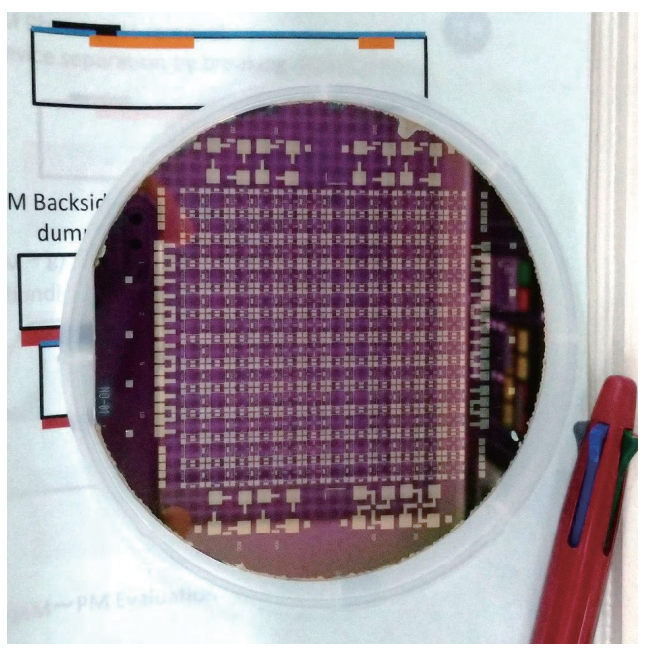

Fig. 4. (Color online) Completely processed 4-inch wafer.

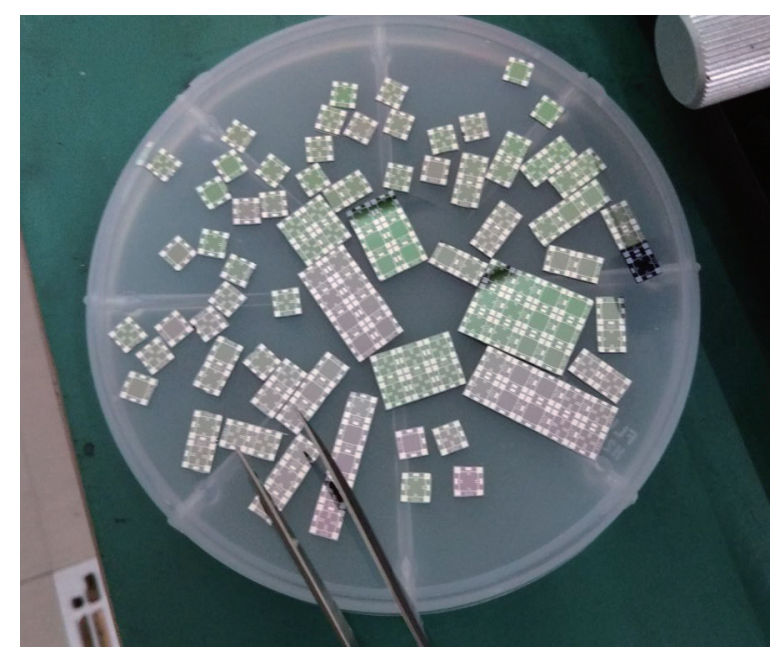

Fig. 5. (Color online) Fabricated piezoresistive force sensor chips.

the trench lines simultaneously formed in the DRIE process (Fig. 5). The MEMS force sensor was attached to a PCB using adhesive and then wire bonding was carried out using a wedge bonder (West Bond). The PCB was double-sided and was designed and fabricated in advance. Finally, a rubber hemisphere was attached to the sensor.

\section{Implementation of IoT Force Sensor and Testing}

\subsection{IoT module implementation}

The electrical circuit shown in Fig. 6 was assembled on the PCB with the fabricated MEMS force sensor. The sensor bridge was driven by a constant current source utilizing an operational amplifier. The output of the sensor bridge was differentially amplified by an instrumentation amplifier. The voltage range of the final output of the circuit was adjusted from 0 to $1 \mathrm{~V}$, which was the acceptable input voltage range of the A/D converter. The completed IoT sensor module is shown in Fig. 7.

To establish an active IoT module, the sensor output was wirelessly transferred to a smartphone or a tablet PC via a WiFi module and the internet, as shown in Fig 8. The analog output of the sensor was connected to the $10 \mathrm{bit}$ A/D converter port of the WiFi module (ESPWROOM-02, Espressif Systems). The core of the WiFi module is an ESP8266 WiFi microchip, which can be programmed similarly to an Arduino microcontroller. A miniature developer board (ESPr Developer, Switch Science), consisting of the ESP8266 WiFi module and its peripheral circuit for the power supply and USB connection, was prepared for this student program. The ESPr Developer was attached to the pin socket on the PCB and was appropriately programmed by a PC through the USB connection, under a development environment common to the Arduino microcontroller. 


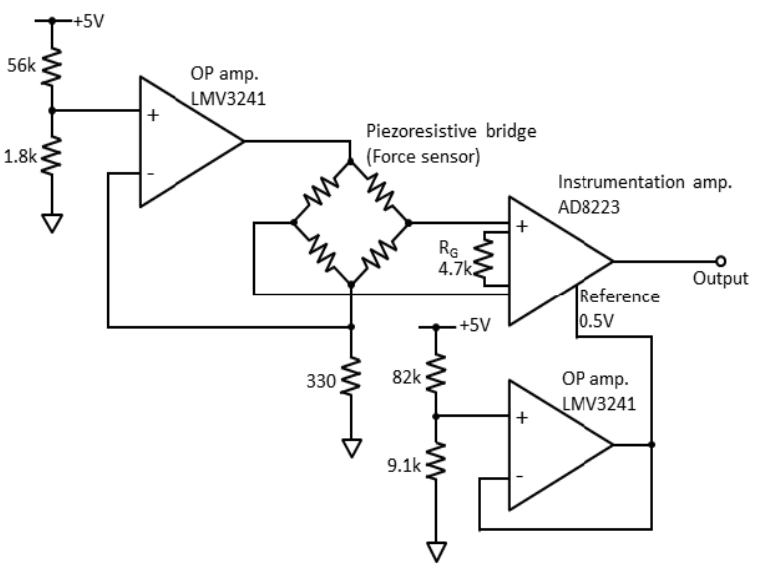

Fig. 6. Electrical circuit for driving piezoresistive bridge and differential amplification.

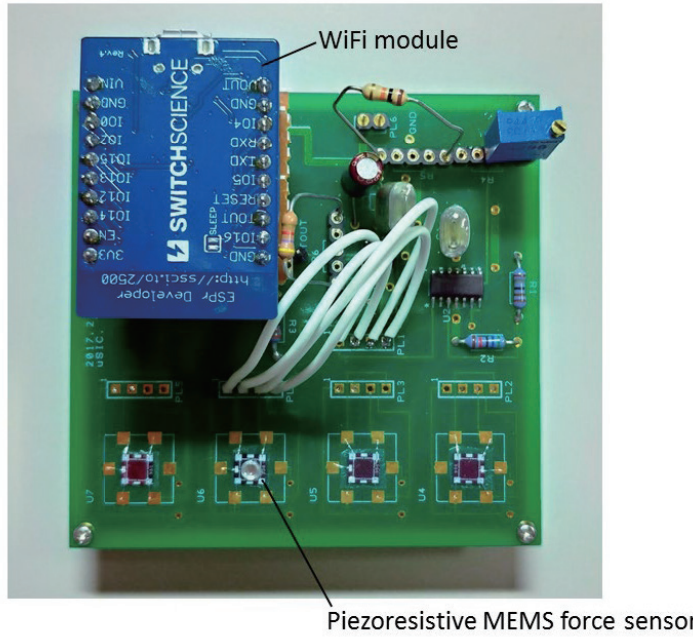

Fig. 7. (Color online) IoT module including MEMS force sensor, WiFi module, and peripheral circuit.

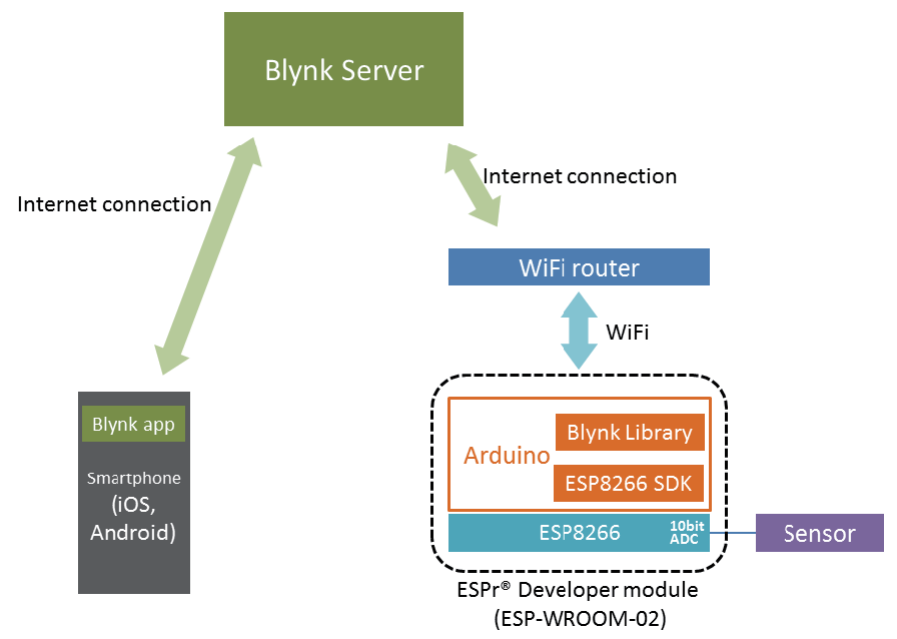

Fig. 8. (Color online) Schematic diagram of IoT system utilizing Blynk platform.

We adopted Blynk as the IoT platform to connect the sensor module to a cloud server that could be easily accessed by smartphones or tablet PCs. ${ }^{(10)}$ The Blynk library was installed in the Arduino microcontroller on the WiFi module. On the other side, the Blynk app was installed in target smartphones or tablet PCs. The Blynk app can access the WiFi module via the internet and the Blynk cloud server. Advantageous features of Blynk are that the installation procedure is relatively simple, a certain capacity can be used free of charge, and the interface is simple to use. Prior to the student practical program, we prepared a sample program to control 
the Arduino microcontroller with the Blynk library. The students installed the program in their WiFi module with a minor modification. An example of a display of the Blynk app on a smartphone connected to the IoT force sensor module is shown in Fig. 9. Changes in the signal can be continuously monitored on the display.

\subsection{Testing}

We tested the IoT force sensor module by applying a force, which was measured by a commercialized force gauge. An example of captured sensor output data is shown in Fig. 10. An applied force ranging from 0 to 220 gf was successfully monitored by the fabricated sensor, where the length and width of the piezoresistive element and the diameter of the diaphragm were $600 \mu \mathrm{m}, 2 \mu \mathrm{m}$, and $3 \mathrm{~mm}$, respectively. The sensitivity of the sensor was $3.6 \mathrm{mV} / \mathrm{gf}$.

\section{Discussion}

In this program, we focused on offering students the opportunity to participate in a shortterm fabrication process of a MEMS force sensor and IoT module implementation in as practical manner as possible. Therefore, the simplified fabrication process of the piezoresistive force sensor was proposed. The process was successfully demonstrated by the students. Before starting this program, we expected that the proposed program would be difficult to complete in

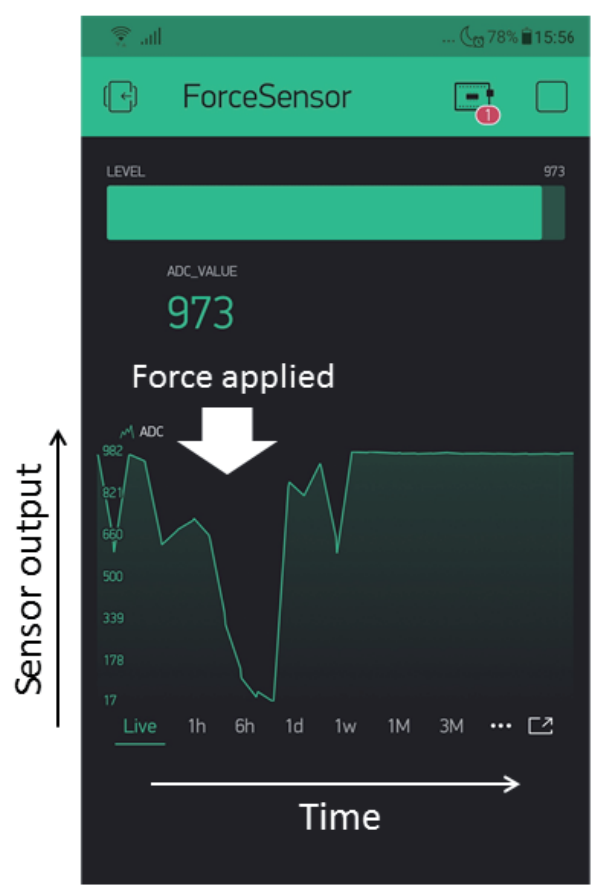

Fig. 9. (Color online) Continuous monitoring of applied force on smartphone display (http://youtu.be/ QakUOerXLt0). 


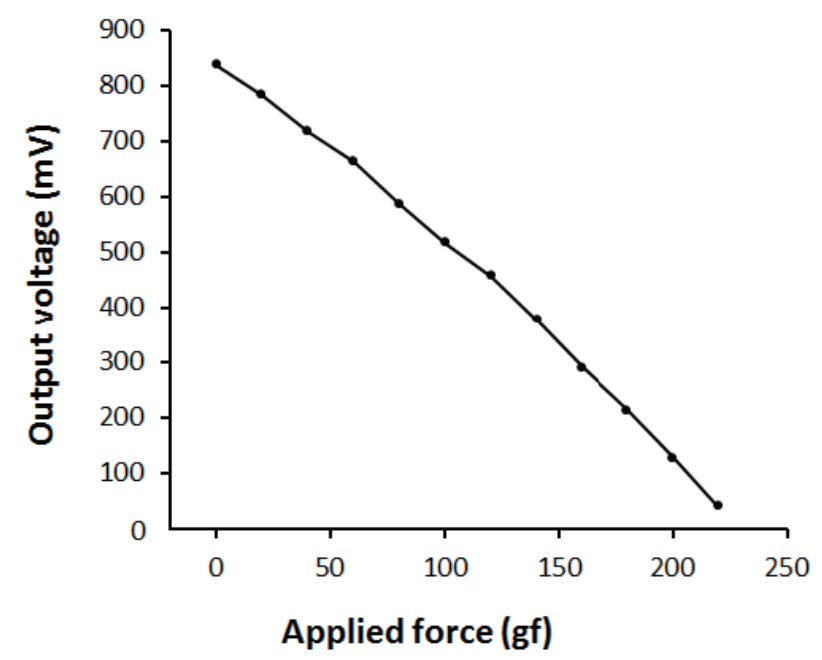

Fig. 10. Sensor output voltage as a function of applied force.

five days. However, all of the participating students successfully completed the whole program in five days and took their fabricated IoT sensor module home. One of the factors contributing to this success is that Nanotechnology Platform Japan including the fabrication facility at Tohoku University has accumulated considerable knowledge of nano-microtechnology and tools over the last decade and offers support through attentive technical staff, who can effectively lead and help students. After completing the program, the students commented that they were able to comprehensively learn about the MEMS fabrication process and the implementation of the IoT application. They also mentioned that such experience and knowledge would stimulate their own research. We concluded that the demonstrated program is valuable for students and engineers attempting to build IoT sensor modules by themselves from sensor fabrication to module implementation. In accordance with requests from other participants in the future, the contents of the program will be modified to provide experience of fabricating other sensors and wireless communication tools.

\section{Conclusions}

We have successfully demonstrated a wireless IoT module including a fabricated piezoresistive MEMS force sensor as a five-day practical program for students at HandsOn-Access Fabrication Facility of Tohoku University, a member of the Nanotechnology Platform Japan project. We found that the resources of the platform effectively support such a challenging program owing to the knowledge and tools accumulated by the platform and the attentive staff. We believe that this practical, short-term program will contribute to accelerating the development of IoT applications utilizing MEMS sensors while nurturing engineers specializing in IoT. 


\section{Acknowledgments}

We thank Mr. Masahiro Ohara from Chiba University, Mr. Naoki Hara from Kagawa University, and Mr. Jianlin Chen from Tohoku University for participating in the program and providing the figures. The program for students was fully supported by Nanotechnology Platform Japan, sponsored by the Ministry of Education, Culture, Sports, Science and Technology (MEXT).

\section{References}

1 Nanotechnology Platform Japan: https://www.nanonet.go.jp/ntj/english/ (accessed April 2019).

2 O. N. Tufte, P. W. Chapman, and D. Long: J. Appl. Phys. 33 (1962) 3322.

3 H. Guckel: Sens. Actuators, A 28 (1991) 133.

4 Z. Chu, P. M. Sarro, and S. Middelhoek: Sens. Actuators, A 54 (1996) 505.

5 A. A. Barlian, W. T. Park, J. R. Mallon, Jr., A. J. Rastegar, and B. L. Pruitt: Proc. IEEE 97 (2009) 513.

6 K. Totsu, M. Moriyama, Y. Suzuki, and M. Esashi: Sens. Mater. 30 (2018) 701.

7 Hands-On-Access Fabrication Facility, Tohoku University: http://www.mu-sic.tohoku.ac.jp/coin_e/index.html (accessed April 2019).

8 Y. Kanda: IEEE Trans. Electron Devices ED-29 (1982) 64.

9 O. N. Tufte and E. L. Stelzer: J. Appl. Phys. 34 (1963) 313.

10 Blynk: https://blynk.io/ (accessed April 2019). 